\title{
Pineal region tumors: an optimal approach for simultaneous endoscopic third ventriculostomy and biopsy
}

\author{
Peter F. Morgenstern, B.A., ${ }^{1}$ Nathan Osbun, B.A., ${ }^{1}$ Theodore H. Schwartz, M.D., ${ }^{1}$ \\ Jeffrey P. Greenfield, M.D., Ph.D., ${ }^{1}$ Apostolos John Tsiouris, M.D., 2 \\ AND Mark M. Souweidane, M.D. ${ }^{1}$
}

Departments of ${ }^{1}$ Neurological Surgery and ${ }^{2}$ Radiology, Weill Cornell Medical College, New York, New York

\begin{abstract}
Object. Simultaneous endoscopic third ventriculostomy (ETV) and tumor biopsy is a widely accepted therapeutic and diagnostic procedure for patients with noncommunicating hydrocephalus secondary to a pineal region tumor. Multiple approaches have been advocated, including the use of a steerable fiberoptic or rigid lens endoscope via 1 or 2 trajectories. However, the optimal approach has not been established based on the individual anatomical characteristics of the patient.

Methods. A retrospective review of patients undergoing simultaneous ETV and tumor biopsy was undertaken. Preoperative MR images were examined to measure the width of the anterior third ventricle and maximal diameters of the tumor, Monro foramen (right), and massa intermedia. The distances between the tumor and massa intermedia, tumor and anterior commissure, midbrain and massa intermedia, and the dorsum sella and anterior commissure were also recorded. Single and dual trajectory approaches were compared using paired t-tests for each parameter.

Results. Over an 8-year interval, 15 patients underwent simultaneous ETV and tumor management. These patients ranged from 6 to 71 years of age (mean 36.7 years); 5 were younger than 18 years of age. Seven were treated using a dual trajectory approach, and 8 were treated using a single trajectory approach. All cases were completed without complications or the need for an additional CSF diversionary procedure within 6 months. The diagnostic yield at biopsy was $86.7 \%$. There were no statistically significant differences between the single and dual trajectory groups for the measured parameters. However, the dual trajectory group demonstrated a larger anterior third ventricular diameter $(1.43 \mathrm{vs} 1.21 \mathrm{~cm}, \mathrm{p}=0.29)$. The single trajectory group trended toward a smaller tumor-anterior commissure interval $(2.23 \mathrm{vs} 2.51 \mathrm{~cm}, \mathrm{p}=0.24)$ and a larger dorsum sella-anterior commissure distance (1.67 vs $1.49 \mathrm{~cm}, \mathrm{p}=0.28)$.

Conclusions. These data confirm the safety and diagnostic efficacy of simultaneous ETV and biopsy for tumors of the pineal region. Although no statistically significant differences were seen in the authors' recorded measurements, several trends suggest a role for a tailored approach to selecting a single or dual trajectory approach when using a rigid endoscope. (DOI: 10.3171/2011.2.FOCUS10301)
\end{abstract}

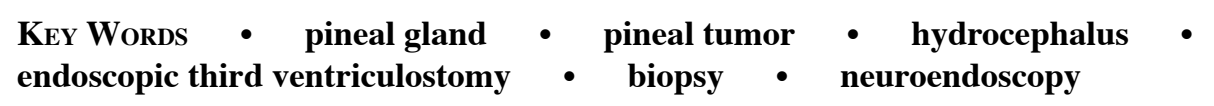

$\mathrm{E}$ NDOSCOPIC biopsy has become a mainstay of the initial approach to tumors in the pineal region. Furthermore, approximately $90 \%$ of patients with these masses present with hydrocephalus requiring management. ${ }^{16,22}$ Ventriculoperitoneal shunting or external ventricular drainage are options for management, but ETV offers a minimally invasive, safe method to treat hydrocephalus resulting from obstruction by the lesion. Endoscopic third ventriculostomy can be performed safely at the time of biopsy, ${ }^{7}$ thus combining therapeutic and diagnostic functions and reducing the total number of procedures for the patient. ${ }^{2,13,15,17}$ While simultaneous ETV and biopsy is now a common management strategy

Abbreviation used in this paper: ETV = endoscopic third ventriculostomy. for tumors in the pineal region, an optimal surgical approach has not been established. In this report, we review our experience with simultaneous ETV and biopsy of tumors in the pineal region and consider potential factors favoring the selection of either a single or dual trajectory approach.

\section{Methods}

Measurements defining the ventricular environment were studied in patients undergoing simultaneous ETV and biopsy of a pineal region mass. A retrospective review of patients between 2002 and 2010 was completed, and 15 patients were identified. Patient data including age, sex, date of surgery, diagnosis, and any associated complications 
were recorded. Complications were defined as the need for shunting in the 6-month period following surgery or any significant perioperative complication recorded in the electronic medical record. The institutional review board of Weill Cornell Medical College approved the review of clinical and radiographic records for this study.

The details of the surgical procedure and equipment used have been described previously. ${ }^{12,19}$ In brief, stereotactic navigational guidance equipment (BrainLAB, Inc.) was used to optimize the planned trajectory and entry sites. Planning an optimal trajectory will reduce motion and torque on the endoscopic path due to sweeping motions of the scope with intraventricular navigation. A $0^{\circ}$ or $30^{\circ}$ rigid lens endoscope (Minop, B. Braun Aesculap) was used for ventricular cannulation. At the time of initial ventricular cannulation, CSF was collected for biochemical and cytological analysis. When performing simultaneous ETV and tumor biopsy, the ETV is performed prior to tumor biopsy. This order is advocated since the most pressing clinical condition (that is, noncommunicating hydrocephalus) should be definitively addressed prior to any potential visual obscuration by hemorrhage from tumor biopsy.

Following successful ETV, biopsy of the tumor was performed. If a single entry site was used, the $30^{\circ}$-angled lens was then rotated to achieve a posterior direction of view. If a separate anterior entry was used, a $0^{\circ}$ lens was used to visualize the posterior third ventricle. In the event that the tumor was eccentric, the approach used a contralateral entry. Once the tumor was visualized, cupped biopsy forceps was used to sample the tumor. Sites of sampling that most likely represented pathological tissue, were relatively void of surface vascularity, and required as little torque as possible were chosen for biopsy. The small samples of tissue obtained with cupped forceps present challenges for accurate pathological interpretation, and every attempt was made to minimize artifact from cautery. Therefore, the use of coagulation on the tumor surface, as logical as that may seem, was avoided prior to sampling. The number of samples was governed by pathological interpretation, and no more tissue than absolutely necessary was taken in an effort to reduce intraventricular hemorrhage.

Magnetic resonance imaging examinations were performed using 1.5-T Signa HDx (8 patients) or 3T Signa HDxt (7 patients) imaging units (General Electric) utilizing a 16-channel-array head coil. In 3 patients, high-resolution coronal T2-weighted fast spin echo images were acquired using the following parameters: TR $6700 \mathrm{msec}$, TE $86 \mathrm{msec}$ with a matrix of $512 \times 512$, and an interleaved 3-mm slice thickness. For 7 patients, 3D T1-weighted spoiled gradient echo (SPGR) images were also acquired following the intravenous administration of gadopentetate dimeglumine (Magnevist, Schering) using the following parameters: TR $8 \mathrm{msec}$, TE minimum (preset by MR imaging software), matrix $256 \times 256$, and 1.5 -mm slice thickness. Measurements acquired on these sequences were confirmed using conventional multiplanar images (Fig. 1). In patients for whom the above images were not available, conventional sequences were used alone.

The diameter of the anterior third ventricle was used as an indicator of the degree of hydrocephalus. Other measurements included the maximal diameters of the tumor, Monro foramen (right), and massa intermedia, as well as the distances between the tumor and massa intermedia, tumor and anterior commissure, midbrain and massa intermedia, and the dorsum sella and anterior commissure. All measurements were performed by a boardcertified neuroradiologist (A.J.T.) on a GE Advantage Workstation v4.3. Single and dual trajectory approaches were compared using paired t-tests for each parameter.

\section{Results}

Patients ranged in age from 6 to 71 years (mean 36.7 years) at the time of surgery; there were 8 male and $7 \mathrm{fe}$ -
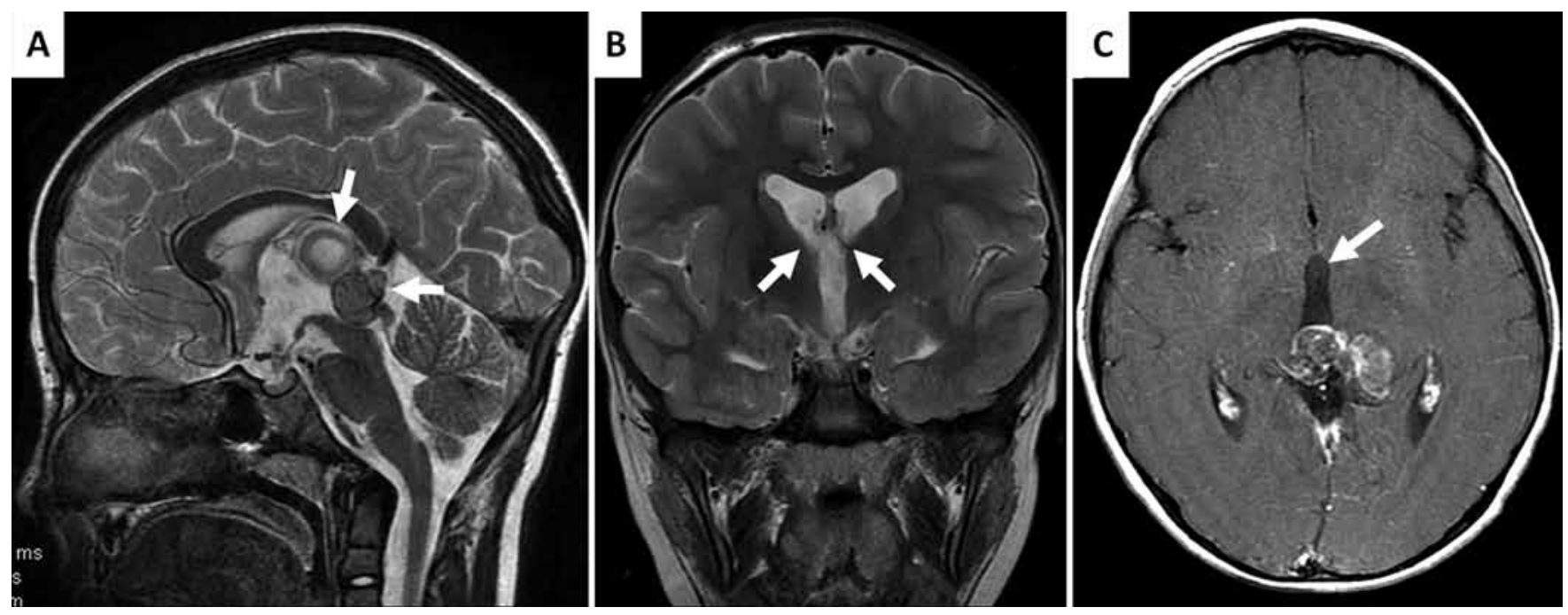

Fig. 1. Multiplanar MR images obtained in a 6-year-old boy presenting with headaches. A: Sagittal T2-weighted image demonstrating a complex mass in the pineal region (arrows). B: Coronal T2-weighted image showing the maximal diameters of the Monro foramina (arrows). C: Axial T1-weighted sequence showing the size of the anterior third ventricle, a measure of the degree of hydrocephalus (arrow). 


\section{Simultaneous ETV and biopsy for pineal region tumors}

TABLE 1: Demographics of patients undergoing simultaneous ETV and pineal mass biopsy

\begin{tabular}{rcclcc}
\hline Case No. & Age (yrs), Sex & $\begin{array}{c}\text { No. of Bur } \\
\text { Holes }\end{array}$ & Diagnosis & $\begin{array}{c}\text { Subsequent } \\
\text { Resection }\end{array}$ & ETV Failure \\
\hline 1 & $57, \mathrm{~F}$ & 1 & pineocytoma & yes & no \\
2 & $59, \mathrm{~F}$ & 1 & pineal cyst & no & no \\
3 & $46, \mathrm{~F}$ & 1 & pineoblastoma & yes & no \\
4 & $17, \mathrm{M}$ & 1 & pineal cyst & no & no \\
5 & $35, \mathrm{~F}$ & 1 & indeterminate & yes & no \\
6 & $71, \mathrm{M}$ & 1 & metastatic adenocarcinoma & no & no \\
7 & $43, \mathrm{~F}$ & 2 & indeterminate* & yes & no \\
8 & $18, \mathrm{M}$ & 2 & germinoma & no & no \\
9 & $30, \mathrm{~F}$ & 2 & pineal parenchymal tumor of intermediate differentiation & yes & no \\
10 & $17, \mathrm{M}$ & 2 & germinoma & no & no \\
11 & $6, \mathrm{M}$ & 1 & mature teratoma & yes & no \\
12 & $15, \mathrm{M}$ & 2 & germinoma & no & no \\
13 & $25, \mathrm{~F}$ & 1 & pineocytoma & yes & no \\
14 & $52, \mathrm{M}$ & 2 & high-grade glioma & yes & no \\
15 & $57, \mathrm{M}$ & 2 & anaplastic astrocytoma & yes & no \\
\hline
\end{tabular}

* Both patients with indeterminate biopsies were diagnosed with pineal parenchymal tumors of indeterminate differentiation after resection.

male patients. The most common diagnosis was germinoma, seen in 3 of the 15 patients. Endoscopic third ventriculostomy and biopsy was performed via 1 or 2 bur holes in 8 and 7 patients, respectively. The diagnostic yield at biopsy was $86.67 \%$ overall. There were no complications following surgery, and the ETV success rate was $100 \%$ in the first 6 months (Table 1).

There were no statistically significant differences between the single and dual trajectory groups for the measured parameters (Table 2). The dual trajectory group, however, trended toward a greater anterior third ventricular diameter (1.43 vs $1.21 \mathrm{~cm}, \mathrm{p}=0.29)$. The single trajectory group trended toward a smaller tumor-anterior commissure interval $(2.23$ vs $2.51 \mathrm{~cm}, \mathrm{p}=0.24)$ and a larger dorsum sella-anterior commissure distance (1.67 vs 1.49 $\mathrm{cm}, \mathrm{p}=0.28)$.

\section{Discussion}

Tumors of the pineal region are rare, comprising less than $11 \%$ of all pediatric tumors. ${ }^{6}$ In children, approximately $60 \%$ of pineal region tumors are of germ cell origin. ${ }^{21}$ In these cases, the presence of $\alpha$-fetoprotein or $\beta$-human chorionic gonadotropin in serum or CSF is sufficient to diagnose malignant germ cell tumors without the need for biopsy. ${ }^{11}$ When these markers are negative, tissue diagnosis becomes necessary, as different histological tumor types have variable responses to radiation therapy, chemotherapy, aggressive resection, or a combination of these treatment modalities. ${ }^{3-5,10}$

\section{Biopsy and Initial Management}

Traditional methods of sampling pineal region masses include microsurgical and stereotactic biopsies. Both approaches result in increased morbidity for a variety of reasons including the vascularity of the pineal region..$^{22}$ Furthermore, recovery and operative time are longer in most cases accomplished via a microsurgical approach. Endoscopic biopsy has been widely described for the management of intraventricular tumors. ${ }^{8,9,14,16,18,20}$ Endoscopy is now the preferred approach to biopsy in this region because it allows for direct visualization, simultaneous treatment of coexisting hydrocephalus, and is diagnostically sensitive ${ }^{18,22}$ and minimally invasive.

Since its description in 1997, simultaneous ETV and biopsy has become an important procedure in the early management of pineal region masses with concurrent hydrocephalus, thus combining therapeutic and diagnostic functions and reducing the total number of procedures for the patient. ${ }^{2,13,15,17}$ The combined procedure also al-

TABLE 2: Parameter measurements of single and dual trajectory approaches to ETV and biopsy

\begin{tabular}{lccc}
\hline & \multicolumn{2}{c}{ Measurement (cm) } & \\
\cline { 2 - 3 } \multicolumn{1}{c}{ Parameter } & $\begin{array}{c}\text { Single } \\
\text { Trajectory }\end{array}$ & $\begin{array}{c}\text { Dual } \\
\text { Trajectory }\end{array}$ & $\mathrm{p}$ \\
(8 patients) & (7 patients) & Value \\
\hline anterior 3rd ventricle diameter & 1.21 & 1.43 & 0.29 \\
Monro foramen diameter (rt) & 0.61 & 0.69 & 0.61 \\
max massa intermedia diameter & 0.51 & 0.43 & 0.50 \\
max tumor diameter & 2.49 & 2.56 & 0.85 \\
tumor-massa distance & 0.81 & 0.90 & 0.61 \\
tumor-anterior commissure dis- & 2.23 & 2.51 & 0.24 \\
$\quad$ tance & & & \\
midbrain-massa distance & 1.10 & 1.12 & 0.90 \\
dorsum sella-anterior commissure & 1.67 & 1.49 & 0.28 \\
$\quad$ distance & & & \\
\hline
\end{tabular}


lows for sampling of CSF for tumor marker assays and examination for tumor dissemination. Multiple studies have demonstrated its safety, diagnostic efficacy, and lower morbidity and mortality compared with conventional approaches. ${ }^{1,2,8,9,13,22}$ Our data support the results of earlier studies, in that our diagnostic efficacy exceeded $80 \%$ with no complications.

\section{Approaches to Simultaneous ETV and Biopsy}

While simultaneous ETV and biopsy is now a common management strategy for tumors in the pineal region, an optimal surgical approach has not been established. Different authors have reported the use of flexible fiberoptic or rigid lens endoscopes through 1 or 2 bur holes with varying degrees of success. Some have recommended the use of a flexible endoscope when combining ETV and biopsy to "avoid damage from moving a less-maneuverable rigid endoscope." ${ }^{13}$ We advocate the use of a rigid lens endoscope because of its superior optical quality, image resolution, and greater size of compatible biopsy forceps. Larger biopsies have been shown to increase the diagnostic yield of the procedure without a measurable increase in morbidity or mortality. ${ }^{1}$

The optimal trajectories for ETV (coronal entry) and pineal region tumor biopsy (frontal-precoronal entry) are distinct, requiring that a single or dual entry site must be chosen if a rod lens endoscope is used. Many approaches are in use today, but it is advisable to choose a technique that best suits the individual patient based on the ventricular size, the relative position of the tumor, the dimension of the massa intermedia, and the surgical goal. It has been suggested that one use a single bur hole but that the bur hole be moved slightly more anteriorly than the typical approach to ETV to accommodate both procedures. ${ }^{16}$ Yurtseven et al. ${ }^{23}$ reported, "For tumors located in the pineal region... we prefer a burr hole just behind the hairy skin line in the midpupillary line," also with a rigid endoscope. Pople et al., ${ }^{15}$ on the other hand, used a dual approach, pointing out that "a separately placed more posterior burrhole was necessary to perform the third ventriculostomy."

Our experience has suggested that a single approach is best used when the tumor presents anterior to the massa intermedia, when the interthalamic adhesion is small, when the degree of ventriculomegaly is severe, or when the surgical intent is biopsy without consideration for total removal. If a single entry is used, a $30^{\circ}$-angled lens is recommended as a means to enhance the view off a linear axis. Alternatively, when tumors are recessed behind the massa intermedia, when the degree of ventriculomegaly is moderate or small, when tumors may be amenable to total removal (that is, those $<2 \mathrm{~cm}$ ), or when the massa intermedia is large, 2 sites of entry are advocated. One should be optimal for the tumor biopsy/resection and the other for the ETV. These features are outlined in Table 3.

\section{Preoperative Planning and Radiographic Measurements}

We have recognized with time that these features may be measurable using preoperative imaging, thereby allowing for a more stereotyped approach to procedure selection. The measurements above are designed to delin-
TABLE 3: Clinical features favoring a dual or single entry approach to simultaneous ETV and biopsy

\begin{tabular}{lccll}
\hline Entry & $\begin{array}{c}\text { Ventricular } \\
\text { Size }\end{array}$ & $\begin{array}{c}\text { Massa } \\
\text { Intermedia }\end{array}$ & $\begin{array}{c}\text { Tumor/Massa } \\
\text { Relationship }\end{array}$ & $\begin{array}{c}\text { Surgical } \\
\text { Goal }\end{array}$ \\
\hline single & large & small & anterior/inferior & biopsy \\
dual & small & large & posterior/superior & removal \\
\hline
\end{tabular}

eate these and other parameters more objectively. While there are no significant differences between the 2 groups in our study, several trends suggest that this radiographically based approach to planning may be valuable.

The use of 1 bur hole was associated with a smaller interval between the anterior commissure and the tumor, a parameter that describes the anterior extent of the tumor. Tumors extending farther forward were more accessible to the surgeon along a trajectory that was also suitable for ETV. The trend toward a greater dorsum sella-anterior commissure distance was also associated with a single trajectory approach and similarly describes a more accessible tumor.

There are several explanations for the absence of a significant difference between the single and dual trajectory groups. Given the range of patient ages and the variance we observed in the data, a more homogeneous group of patients may limit the variance and allow more minute differences to be detected between the groups. Similarly, a larger patient sample may ultimately allow the data to achieve statistical significance. It also may be that either approach is appropriate for any patient, and that the consequences of selecting a suboptimal approach are too minor to be observed clinically. Furthermore, there may be other metrics that differ between the groups that we have not considered, such as degree of intraventricular hemorrhage or forniceal contusion. Despite the lack of statistical significance in this small patient sample, the parameters we have described appear to be useful in determining whether the tumor can be targeted for biopsy along the same trajectory by which one would be able to access the floor of the third ventricle for ETV. Using measurements to choose a single or dual bur hole approach could aid clinical judgment and avoid the potential risk of introducing a second trajectory when it is unnecessary.

\section{Conclusions}

Our data confirm the safety and diagnostic efficacy of simultaneous ETV and biopsy in the early management of a newly diagnosed pineal region mass. We have also demonstrated that, while it is not yet possible to recommend a set of standard criteria requiring one approach over another, several of our measurements showed trends toward a particular approach. Further evaluation of a larger patient cohort may allow us to make such recommendations.

\section{Disclosure}

Dr. Souweidane serves as a paid consultant and member of the NeuroEndoscopy Advisory Board for Aesculap.

Author contributions to the study and manuscript prepara- 


\section{Simultaneous ETV and biopsy for pineal region tumors}

tion include the following. Conception and design: Souweidane, Morgenstern, Osbun. Acquisition of data: Morgenstern, Osbun, Schwartz, Greenfield, Tsouris. Analysis and interpretation of data: Souweidane, Morgenstern, Osbun, Tsouris. Drafting the article: Souweidane, Morgenstern, Osbun, Tsouris. Critically revising the article: Souweidane, Morgenstern, Osbun, Tsouris. Reviewed final version of the manuscript and approved it for submission: Souweidane, Schwartz, Greenfield, Tsouris. Statistical analysis: Morgenstern. Administrative/technical/material support: Morgenstern. Study supervision: Souweidane.

\section{References}

1. Ahn ES, Goumnerova L: Endoscopic biopsy of brain tumors in children: diagnostic success and utility in guiding treatment strategies. Clinical article. J Neurosurg Pediatr 5:255262,2010

2. Al-Tamimi YZ, Bhargava D, Surash S, Ramirez RE, Novegno F, Crimmins DW, et al: Endoscopic biopsy during third ventriculostomy in paediatric pineal region tumours. Childs Nerv Syst 24:1323-1326, 2008

3. Cho BK, Wang KC, Nam DH, Kim DG, Jung HW, Kim HJ, et al: Pineal tumors: experience with 48 cases over 10 years. Childs Nerv Syst 14:53-58, 1998

4. Czirják S, Vajda J, Pásztor E: Management of pineal region tumours. Neurol Res 14:241-247, 1992

5. Dhall G, Khatua S, Finlay JL: Pineal region tumors in children. Curr Opin Neurol 23:576-582, 2010

6. Drummond KJ, Rosenfeld JV: Pineal region tumours in childhood. A 30-year experience. Childs Nerv Syst 15:119-127, 1999

7. Ellenbogen RG, Moores LE: Endoscopic management of a pineal and suprasellar germinoma with associated hydrocephalus: technical case report. Minim Invasive Neurosurg 40: 13-16, 1997

8. Ferrer E, Santamarta D, Garcia-Fructuoso G, Caral L, Rumià $\mathrm{J}$ : Neuroendoscopic management of pineal region tumours. Acta Neurochir (Wien) 139:12-21, 1997

9. Gangemi M, Maiuri F, Colella G, Buonamassa S: Endoscopic surgery for pineal region tumors. Minim Invasive Neurosurg 44:70-73, 2001

10. Kang JK, Jeun SS, Hong YK, Park CK, Son BC, Lee IW, et al: Experience with pineal region tumors. Childs Nerv Syst 14:63-68, 1998

11. Luther N, Edgar MA, Dunkel IJ, Souweidane MM: Correlation of endoscopic biopsy with tumor marker status in primary intracranial germ cell tumors. J Neurooncol 79:45-50, 2006

12. Luther N, Stetler WR Jr, Dunkel IJ, Christos PJ, Wellons JC III, Souweidane MM: Subarachnoid dissemination of intraventricular tumors following simultaneous endoscopic biopsy and third ventriculostomy. Clinical article. J Neurosurg Pediatr 5:61-67, 2010
13. O'Brien DF, Hayhurst C, Pizer B, Mallucci CL: Outcomes in patients undergoing single-trajectory endoscopic third ventriculostomy and endoscopic biopsy for midline tumors presenting with obstructive hydrocephalus. J Neurosurg 105 (3 Suppl):219-226, 2006

14. Oi S, Shibata M, Tominaga J, Honda Y, Shinoda M, Takei F, et al: Efficacy of neuroendoscopic procedures in minimally invasive preferential management of pineal region tumors: a prospective study. J Neurosurg 93:245-253, 2000

15. Pople IK, Athanasiou TC, Sandeman DR, Coakham HB: The role of endoscopic biopsy and third ventriculostomy in the management of pineal region tumours. Br J Neurosurg 15: 305-311, 2001

16. Robinson S, Cohen AR: The role of neuroendoscopy in the treatment of pineal region tumors. Surg Neurol 48:360-367, 1997

17. Shono T, Natori Y, Morioka T, Torisu R, Mizoguchi M, Nagata $\mathrm{S}$, et al: Results of a long-term follow-up after neuroendoscopic biopsy procedure and third ventriculostomy in patients with intracranial germinomas. J Neurosurg 107 (3 Suppl):193-198, 2007

18. Song JH, Kong DS, Shin HJ: Feasibility of neuroendoscopic biopsy of pediatric brain tumors. Childs Nerv Syst 26:15931598,2010

19. Souweidane MM, Morgenstern PF, Kang S, Tsiouris AJ, Roth J: Endoscopic third ventriculostomy in patients with a diminished prepontine interval. Clinical article. J Neurosurg Pediatr 5:250-254, 2010

20. Souweidane MM, Sandberg DI, Bilsky MH, Gutin PH: Endoscopic biopsy for tumors of the third ventricle. Pediatr Neurosurg 33:132-137, 2000

21. Weiner HL, Finlay JL: Surgery in the management of primary intracranial germ cell tumors. Childs Nerv Syst 15:770-773, 1999

22. Yamini B, Refai D, Rubin CM, Frim DM: Initial endoscopic management of pineal region tumors and associated hydrocephalus: clinical series and literature review. J Neurosurg 100 (5 Suppl Pediatrics):437-441, 2004

23. Yurtseven T, Erşahin Y, Demirtaş E, Mutluer S: Neuroendoscopic biopsy for intraventricular tumors. Minim Invasive Neurosurg 46:293-299, 2003

Manuscript submitted December 14, 2010.

Accepted February 1, 2011.

Portions of this work were presented as an abstract at the International Symposium on Pediatric Neuro-Oncology, Vienna, Austria, June 23, 2010.

Address correspondence to: Mark M. Souweidane, M.D., 525 East 68th Street, Starr Pavilion 651, New York, New York 10065. email: mmsouwei@med.cornell.edu. 